\section{Re: Interventions to Improving Osteoporosis Screening: An Iowa Research Network (IRENE) Study}

To the Editor: In a busy clinical practice with complicated patients, it can be difficult remembering to perform all the recommended preventative care interventions. ${ }^{1}$ Previous studies have demonstrated how the use of prompts improves physician performance of preventative care measures. ${ }^{2}$ The IRENE study uses a simple "sticky note" on patients' charts, with or without patient education, in an effort to improve rates of bone mineral density (BMD) testing for osteoporosis screening. ${ }^{3}$ The study concludes that the addition of their intervention improved osteoporosis screening within family medicine practices as compared with no intervention in the usual care physician practices. This conclusion may be overestimated given that the baseline characteristics of the intervention and usual care practices were so different.

The usual care physician practice had (1) lower rates of previous BMD screening, (2) fewer numbers of previous annual visits for patient participants, and (3) lower patient educational levels. It would be interesting to investigate other demographic and socioeconomic characteristics of the usual care practice and how those characteristics may have impacted rates of osteoporosis screening in that population. Previous studies of screening for other disease entities, namely cervical cancer ${ }^{4}$ and colon cancer, ${ }^{5}$ have suggested an association between lower socioeconomic indicators (income, education level) and lower screening rates. It may follow that the socioeconomic characteristics of the usual care practice served as significant confounding variables in this study.

In randomized controlled trials, it is important that the intervention and control groups are as similar as possible. In this study, the dissimilarities between the baseline characteristics of the physician practices were significant enough that the validity of the results must be called into question.

Lora A. Cox, MD Department of Family Medicine UPMC St. Margaret Pittsburgh, PA coxla@upmc.edu

\section{References}

1. Yarnall KSH, Pollak KI, Ostbye T, Krause KM, Michener JL. Primary care: Is there enough time for prevention? Am J Public Health 2003;93:635-41.

2. Wallis DH, Chin JL, Sur DK, Lee MY. Increasing rates of influenza vaccination during pregnancy: A multisite interventional study. J Am Board Fam Med 2006;19:345-9.

3. Levy BT, Hartz A, Woodworth G, Xu Y, Sinift S. Interventions to improving osteoporosis screening: An Iowa Re- search Network (IRENE) study. J Am Board Fam Med 2009;22:360-7.

4. Carlos RC, Fendrick AM, Patterson SK, Bernstein SJ. Associations in breast and colon cancer screening behavior in women. Acad Radiol 2005;12(4):451-8.

5. Zimmerman RK, Nowalk MP, Tabbarah M, Grufferman S. Predictors of colorectal cancer screening in diverse primary care practices. BMC Health Serv Res 2006;6:116.

doi: 10.3122/jabfm.2010.01.090200

The above letter was referred to the authors of the article in question, who offer the following reply.

\section{Response: Re: Interventions to Improving Osteoporosis Screening: an Iowa Research Network (IRENE) Study}

To the Editor: Dr. Cox $^{1}$ was concerned that confounding factors might have invalidated this study. ${ }^{2}$ Certainly it is probable that at baseline, before any intervention, some clinics will be more likely than others to refer patients for bone density testing. To some extent we took that into account by adjusting for patient educational achievement, baseline rates of testing at each clinic, and other factors. Statistical testing also takes into account nonmeasured intrinsic patient and clinic factors which contribute to variation among the clinics. Because there were only 5 clinics in the study, there was a high bar for finding differences among the intervention groups beyond what might be due to intrinsic factors. Our results suggested that clinic variation was influenced by the combined intervention of chart reminder to physician plus mailed education to patients. The large improvement in the clinics receiving this combined intervention was striking compared with no change for the control clinic. Because the clinics that received the combined intervention were doing well at baseline, it might be anticipated that they would have improved little without the intervention or even declined.

This is a small study, and we cannot state definitely that the interventions tested were helpful. However, we tried to interpret the data fairly using available statistical methods.

Barcey T. Levy, PhD, MD Department of Family Medicine University of Iowa Carver College of Medicine Iowa City, IA barcey-levy@uiowa.edu

Arthur Hartz, MD, PhD Department of Internal Medicine University of Utah School of Medicine Salt Lake City, UT 
Yinghui Xu, MS

Department of Family Medicine

University of Iowa

Carver College of Medicine

Iowa City, IA

\section{References}

1. Cox LA. Re: Interventions to improving osteoporosis screening: an Iowa Research Network (IRENE) study. J Am Board Fam Med 2010;23:131.

2. Levy BT, Hartz A, Woodworth G, Xu Y, Sinift S. Interventions to improving osteoporosis screening: an Iowa Research Network (IRENE) study. J Am Board Fam Med 2009;22:360-7.

doi: 10.3122/jabfm.2010.01.090216

\section{Re: Interventions to Improving Osteoporosis Screening: An Iowa Research Network (IRENE) Study}

To the Editor: I read with great interest the original research article, "Interventions to Improving Osteoporosis Screening: An Iowa Research Network (IRENE) Study"1 in the July/August 2009 edition of the Fournal of the American Board of Family Medicine. Nationally, the current screening rate for osteoporosis is unacceptably low, and Dr. Levy and her colleagues conducted a welldesigned study to evaluate the effect of 2 simple strategies to improve screening rates. This is a timely issue given the current political debate about the most costeffective way to improve health care delivery. Their study found that chart reminders to physicians did not significantly increase the rate of bone mineral density testing when compared with usual care but that combining chart reminders with a patient-directed mail campaign did significantly increase the rate.

Given that the National Osteoporosis Foundation guidelines recommend bone mineral density testing for all women over the age of 65 , I am curious about one element of Dr. Levy's study design. ${ }^{2}$ In the study, chart reminders were placed on the charts of women older than 65 only when they were being seen for an annual examination. Why not place the chart reminder on the charts of all women older than 65 regardless of the reason for their visit?

By limiting preventive care interventions to scheduled annual exams, family physicians miss opportunities to improve the care of their patients. "Max-packing" is a relatively new concept which refers to the practice of doing as much as possible for patients every time they are in the office. ${ }^{3}$ One way to do this is to identify and address preventive care needs at every office visit. To excel at providing preventive care, family physicians must develop systems that assess needs and prompt delivery of care at every opportunity, not just during annual exams.

Elisa Payne, MD

Area Health Education Center-Fort Smith Family Medicine Residency Program University of Arkansas for Medical Sciences

Fort Smith, AR

EMPayne@uams.edu

\section{References}

1. Levy BT, Hartz A, Woodworth G, Xu Y, Sinift S. Interventions to improving osteoporosis screening: an Iowa Research Network (IRENE) study. J Am Board Fam Med 2009;22:360-7.

2. National Osteoporosis Foundation. Clinician's Guide to Prevention and Treatment of Osteoporosis. Washington, DC: National Osteoporosis Foundation; 2008.

3. Institute for Healthcare Improvement website. Available at www.ihi.org/IHI/Topics?OfficePractices/Access/Changes/ IndividualChanges/UseMaxPackingDuringtheVisit.html. Accessed September 23, 2009.

doi: $10.3122 / \mathrm{jabfm} .2010 .01 .090224$

The above letter was referred to the authors of the article in question, who offer the following reply.

\section{Response: Re: Interventions to Improving Osteoporosis Screening: an Iowa Research Network (IRENE) Study}

To the Editor: Dr. Payne ${ }^{1}$ asked why we did not place reminders on the charts of all women over the age of 65 , regardless of the reason for their visit. ${ }^{2}$ We chose to only recruit women who had an upcoming visit for an annual examination because the annual examination allows extra time to systematically review and address multiple preventive issues. ${ }^{3-5}$ Providing all preventive services and counseling for all patients would take an average of 7.4 hours per working day, ${ }^{6}$ and thus would be impractical. Asking physicians to discuss osteoporosis screening and to provide counseling about bone health at times other than a scheduled preventive visit would place an unfair burden on the busy physicians who volunteered for this study. Even if reminders on all charts would increase response rates for osteoporosis testing, they would probably interfere with other care the patients should receive. A key aspect of a medical home is the systematic tracking and registry function that organize clinical information and remind physicians and patients of services needed. ${ }^{7,8}$ It is clear that the nation needs fundamental payment reforms in primary care to achieve population health.'

Barcey T. Levy, PhD, MD Department of Family Medicine University of Iowa Carver College of Medicine Iowa City, IA barcey-levy@uiowa.edu 